Supporting Information (SI)

\title{
Screening of Transition-Metal Single-Atom Catalysts Anchored on Covalent-Organic Frameworks for Efficient Nitrogen Fixation
}

Juan Wang, Zhihua Zhang, Yangyang Li*, Yuanyuan Qu, Yongqiang Li, Weifeng Li,

Mingwen Zhao*

School of Physics \& State Key Laboratory of Crystal Materials, Shandong

University,Jinan 250100, Shandong, China

*Corresponding author.

E-mail: sduyyl@outlook.com (YL); zmw@sdu.edu.cn (MZ) 
Table S1. The total energy $(E)$, the zero-point energy $\left(E_{\mathrm{ZPE}}\right)$, entropy $\left(T^{*} S\right)$, and free energy of gaseous $\mathrm{N}_{2}, \mathrm{H}_{2}$ and $\mathrm{NH}_{3}$.

\begin{tabular}{ccccc}
\hline Species & $E(\mathrm{eV})$ & $E_{\mathrm{ZPE}}(\mathrm{eV})$ & $\boldsymbol{T} * \boldsymbol{S}(\mathrm{eV})$ & $\Delta G(\mathrm{eV})$ \\
\hline $\mathbf{N}_{\mathbf{2}}$ & -16.63 & 0.15 & 0.62 & -17.07 \\
$\mathbf{H}_{2}$ & -6.78 & 0.27 & 0.40 & -6.90 \\
$\mathbf{N H}_{3}$ & -19.54 & 0.91 & 0.59 & -19.22 \\
\hline
\end{tabular}


Table S2. Thermal Corrections of zero-point energy $\left(E_{\mathrm{ZPE}}\right)$, enthalpy correction $\left(\int C_{p} d T\right)$, and entropy correction $(T \Delta S)$ of different adsorbed species. The entropy correction of the gas-phase molecules was obtained from the NIST database. ${ }^{1}$

\begin{tabular}{|c|c|c|c|}
\hline Species & $E_{\mathrm{ZPE}}(\mathrm{eV})$ & $\int C_{p} d T$ & $T \Delta S$ \\
\hline$\overline{\mathbf{H}_{2}{ }^{1}}$ & 0.27 & 0.09 & 0.40 \\
\hline $\mathbf{N}_{2}{ }^{1}$ & 0.15 & 0.09 & 0.59 \\
\hline $\mathbf{N H}_{3}{ }^{1}$ & 0.89 & 0.11 & 0.60 \\
\hline$* \mathbf{N N}$ & 0.21 & 0.07 & 0.15 \\
\hline *NNH & 0.48 & 0.08 & 0.15 \\
\hline *NNHH & 0.80 & 0.08 & 0.18 \\
\hline$* \mathbf{N}$ & 0.09 & 0.03 & 0.06 \\
\hline *NH & 0.36 & 0.05 & 0.08 \\
\hline$* \mathbf{N H}_{2}$ & 0.69 & 0.06 & 0.09 \\
\hline$* \mathbf{N H}_{3}$ & 1.04 & 0.06 & 0.15 \\
\hline *NHNH & 0.82 & 0.07 & 0.12 \\
\hline$* \mathbf{N H N H}_{2}$ & 1.14 & 0.10 & 0.19 \\
\hline$* \mathbf{N H}_{2} \mathbf{N H}_{2}$ & 1.50 & 0.10 & 0.22 \\
\hline$* \mathbf{N} * \mathbf{N}$ & 0.20 & 0.05 & 0.10 \\
\hline$* \mathbf{N} * \mathbf{N H}$ & 0.49 & 0.07 & 0.11 \\
\hline${ }^{*} \mathbf{N H}{ }^{*} \mathbf{N H}$ & 0.78 & 0.09 & 0.17 \\
\hline$* \mathbf{N} * \mathbf{N H}_{2}$ & 0.85 & 0.07 & 0.14 \\
\hline$* \mathbf{N H} * \mathbf{N H}_{2}$ & 1.14 & 0.10 & 0.21 \\
\hline$* \mathrm{NH}_{2} * \mathbf{N H}_{2}$ & 1.48 & 0.10 & 0.16 \\
\hline
\end{tabular}

$\Delta G=\Delta E+\Delta E_{\mathrm{ZPE}}+\int C_{p} d T-T \Delta S-\Delta G_{U}+\Delta G_{p H}$ 
Table S3. The formation energy $E_{f}$, standard dissolution potentials $\left(U^{\circ}\right.$ diss, $\left.p H=0\right)$, number of electrons $\left(n_{e}\right)$ involved in the dissolution for the bulk metals, and computed dissolution potentials $\left(U_{\text {diss }} /(\mathrm{V}\right.$ vs. SHE) $)$ of TMPc-TFPN. Experimental values for $U^{\circ}$ diss are taken from references. ${ }^{2}$

\begin{tabular}{|c|c|c|c|c|}
\hline TM & $E_{f}(\mathrm{eV})$ & $n_{\mathrm{e}}$ & $U^{\circ} \operatorname{diss}(\mathbf{V})$ & $U_{\text {diss }}(\mathbf{V})$ \\
\hline Sc & -6.37013 & 3 & -2.08 & 0.043375 \\
\hline $\mathbf{T i}$ & -5.32661 & 2 & -1.63 & 1.033303 \\
\hline $\mathbf{V}$ & -4.75408 & 2 & -1.18 & 1.197038 \\
\hline $\mathrm{Cr}$ & -5.42181 & 2 & -0.91 & 1.800906 \\
\hline Mn & -5.33179 & 2 & -1.19 & 1.475896 \\
\hline $\mathrm{Fe}$ & -4.77501 & 2 & -0.45 & 1.937503 \\
\hline Co & -4.67022 & 2 & -0.28 & 2.055108 \\
\hline $\mathbf{N i}$ & -4.95332 & 2 & -0.26 & 2.216660 \\
\hline $\mathbf{C u}$ & -3.79260 & 2 & 0.34 & 2.236300 \\
\hline $\mathbf{Z n}$ & -4.81633 & 2 & -0.76 & 1.648167 \\
\hline $\mathbf{Y}$ & -4.75056 & 3 & -2.37 & -0.786480 \\
\hline $\mathbf{Z r}$ & -4.08370 & 4 & -1.45 & -0.429080 \\
\hline $\mathbf{N b}$ & -2.70053 & 3 & -1.10 & -0.199820 \\
\hline Mo & -2.82413 & 3 & -0.20 & 0.741376 \\
\hline $\mathbf{R u}$ & -2.98942 & 3 & 0.46 & 1.456472 \\
\hline $\mathbf{R h}$ & -3.90711 & 2 & 0.60 & 2.553553 \\
\hline $\mathbf{P b}$ & -4.47980 & 2 & 0.95 & 3.189901 \\
\hline Ag & -2.02441 & 1 & 0.80 & 2.824415 \\
\hline Hf & -4.56818 & 4 & -1.55 & -0.407950 \\
\hline $\mathbf{T a}$ & -3.35332 & 3 & -0.60 & 0.517773 \\
\hline $\mathbf{W}$ & -2.19371 & 2 & 0.10 & 1.196853 \\
\hline $\mathbf{R e}$ & -2.17342 & 3 & 0.30 & 1.024472 \\
\hline Os & -1.98665 & 8 & 0.84 & 1.088332 \\
\hline Ir & -3.64852 & 3 & 1.16 & 2.376172 \\
\hline $\mathbf{P t}$ & -4.79079 & 2 & 1.18 & 3.575395 \\
\hline $\mathbf{A u}$ & -2.33813 & 3 & 1.50 & 2.279377 \\
\hline
\end{tabular}


Table S4. Calculated limiting potential $\left(U_{\mathrm{L}}\right)$ for $\mathrm{NRR}$ on different $\mathrm{TMN}_{4}$ catalysts.

\begin{tabular}{ccc}
\hline Catalysts & $\boldsymbol{U}_{\boldsymbol{L}}(\mathbf{V})$ & Reference \\
\hline Tc $_{3}\left(\mathbf{H A B}_{\mathbf{2}}\right.$ & -0.63 & 3 \\
Ti-TCNQ & -0.47 & 4 \\
Mo- PP & -0.22 & 5 \\
Mo-COF & -0.28 & 6 \\
Mo-Pp & -0.58 & 7 \\
Mo-Pc & -0.70 & 8 \\
Mo-Pc & -0.25 & 9 \\
MoPc & -0.33 & 10 \\
Ti@N & -0.69 & 11 \\
Mo-rTCNQ & -0.29 & 12 \\
MoPc-TFPN & -0.21 & This work \\
WPc-TFPN & -0.19 & This work \\
TaPc-TFPN & -0.21 & This work \\
RePc-TFPN & 0.40 & This work \\
\hline
\end{tabular}


Table S5. Gibbs free energy change of each elementary step for considered TMPcTFPN (Mo, Ta, W, Re) with $\mathrm{N}_{2}$ end-on configuration along distal pathway.

\begin{tabular}{lcccc}
\hline Reaction paths & $\mathbf{M o}(\mathbf{e V})$ & $\mathbf{T a}(\mathrm{eV})$ & $\mathbf{W}(\mathrm{eV})$ & $\mathbf{R e}(\mathrm{eV})$ \\
\hline$*+\mathbf{N}_{\mathbf{2}} \rightarrow \mathbf{N N}$ & -0.57 & -0.94 & -0.76 & -0.87 \\
$*+\mathbf{N}_{\mathbf{2}}+\mathbf{H} \rightarrow * \mathbf{N N H}$ & -0.36 & -0.78 & -0.78 & -0.47 \\
$*+\mathbf{N}_{\mathbf{2}}+\mathbf{2} \mathbf{H} \rightarrow * \mathbf{N N H H}$ & -0.36 & -1.21 & -0.99 & -0.46 \\
$*+\mathbf{N}_{\mathbf{2}}+\mathbf{3 H} \rightarrow * \mathbf{N}+\mathbf{N H}_{\mathbf{3}}$ & -1.49 & -1.53 & -1.92 & -1.83 \\
$*+\mathbf{N}_{\mathbf{2}}+\mathbf{4 H} \rightarrow * \mathbf{N H}+\mathbf{N H}_{\mathbf{3}}$ & -1.56 & -2.61 & -2.29 & -1.60 \\
$*+\mathbf{N}_{\mathbf{2}}+\mathbf{5} \mathbf{H} \rightarrow * \mathbf{N H} \mathbf{H}_{\mathbf{2}}+\mathbf{N H}_{\mathbf{3}}$ & -1.87 & -2.92 & -2.51 & -1.90 \\
$*+\mathbf{N}_{\mathbf{2}}+\mathbf{6 H} \rightarrow * \mathbf{N H}_{\mathbf{3}}+\mathbf{N H}_{\mathbf{3}}$ & -2.12 & -2.71 & -2.32 & -2.23 \\
$*+\mathbf{N}_{\mathbf{2}}+\mathbf{6 H} \rightarrow *+\mathbf{2 N H}_{\mathbf{3}}$ & -0.87 & -0.87 & -0.87 & -0.87 \\
\hline
\end{tabular}


Table S6. The TM-N bond length, the $\mathrm{N} \equiv \mathrm{N}$ bond length, TM-N ICOHP, N $\equiv \mathrm{N}$ ICOHP and the Gibbs free energy change $\left(\Delta G *_{\mathrm{N}_{2}}\right)$ on TMPc-TFPN with end on configurations.

\begin{tabular}{|c|c|c|c|c|c|}
\hline TM & $\begin{array}{c}\text { TM-N band } \\
\text { length }(\AA)\end{array}$ & $\begin{array}{l}\mathrm{N} \equiv \mathrm{N} \text { band } \\
\text { length }(\AA)\end{array}$ & $\begin{array}{c}\text { TM-N } \\
\text { ICOHP }\end{array}$ & $\begin{array}{c}\mathrm{N} \equiv \mathrm{N} \\
\text { ICOHP }\end{array}$ & $\Delta G_{* \mathrm{~N}_{2}}(\mathrm{eV})$ \\
\hline Sc & 2.285 & 1.126 & -0.373 & -8.018 & 0.144 \\
\hline Ti & 2.042 & 1.134 & -1.029 & -7.153 & -0.171 \\
\hline $\mathbf{V}$ & 1.903 & 1.135 & -1.472 & -7.744 & -0.212 \\
\hline $\mathrm{Cr}$ & 1.861 & 1.135 & -1.426 & -7.691 & -0.259 \\
\hline $\mathrm{Fe}$ & 1.737 & 1.135 & -2.231 & -7.678 & -0.499 \\
\hline Co & 1.944 & 1.122 & -0.815 & -8.555 & -0.040 \\
\hline Mo & 1.943 & 1.141 & -3.024 & -7.052 & -0.572 \\
\hline $\mathbf{R u}$ & 1.846 & 1.136 & -3.420 & -7.153 & -0.690 \\
\hline $\mathbf{T a}$ & 2.002 & 1.148 & -2.834 & -6.773 & -0.983 \\
\hline $\mathbf{W}$ & 1.921 & 1.148 & -3.512 & -6.426 & -0.755 \\
\hline $\operatorname{Re}$ & 1.876 & 1.146 & -3.784 & -6.489 & -0.871 \\
\hline Os & 1.836 & 1.143 & -4.078 & -6.451 & -0.957 \\
\hline
\end{tabular}


Table S7. Valance electron in d-orbital electrons $\left(N_{d}\right)$, electronegativity of single TM atom $\left(E_{\mathrm{TM}}\right), \varphi$, the Bader charge variation of $\mathrm{N}_{2}$ and theoretical limiting potential $\left(U_{\mathrm{L}}\right)$ for NRR on TMPc-TFPN.

\begin{tabular}{|c|c|c|c|c|c|}
\hline TM & $N_{d}$ & $E_{\mathrm{TM}}(\mathrm{eV})$ & $\varphi$ & Bader charge & $\mathbf{U}_{\mathrm{L}}(\mathrm{V})$ \\
\hline $\mathrm{Sc}$ & 1 & 1.36 & 0.857 & 0.165 & 1.31 \\
\hline $\mathrm{Ti}$ & 2 & 1.54 & 1.612 & 0.235 & 1.24 \\
\hline $\mathrm{V}$ & 3 & 1.63 & 2.350 & 0.228 & 0.74 \\
\hline $\mathrm{Cr}$ & 5 & 1.66 & 3.881 & 0.228 & 0.56 \\
\hline $\mathrm{Fe}$ & 6 & 1.83 & 4.435 & 0.218 & 1.03 \\
\hline $\mathrm{Co}$ & 7 & 1.88 & 5.105 & 0.117 & 1.90 \\
\hline Mo & 5 & 2.16 & 3.402 & 0.283 & 0.21 \\
\hline $\mathrm{Ru}$ & 7 & 2.20 & 4.719 & 0.213 & 1.30 \\
\hline $\mathrm{Ta}$ & 3 & 1.50 & 2.449 & 0.410 & 0.21 \\
\hline W & 4 & 2.36 & 2.604 & 0.355 & 0.19 \\
\hline $\mathrm{Re}$ & 5 & 1.90 & 3.627 & 0.342 & 0.40 \\
\hline Os & 6 & 2.20 & 4.045 & 0.288 & 1.19 \\
\hline
\end{tabular}




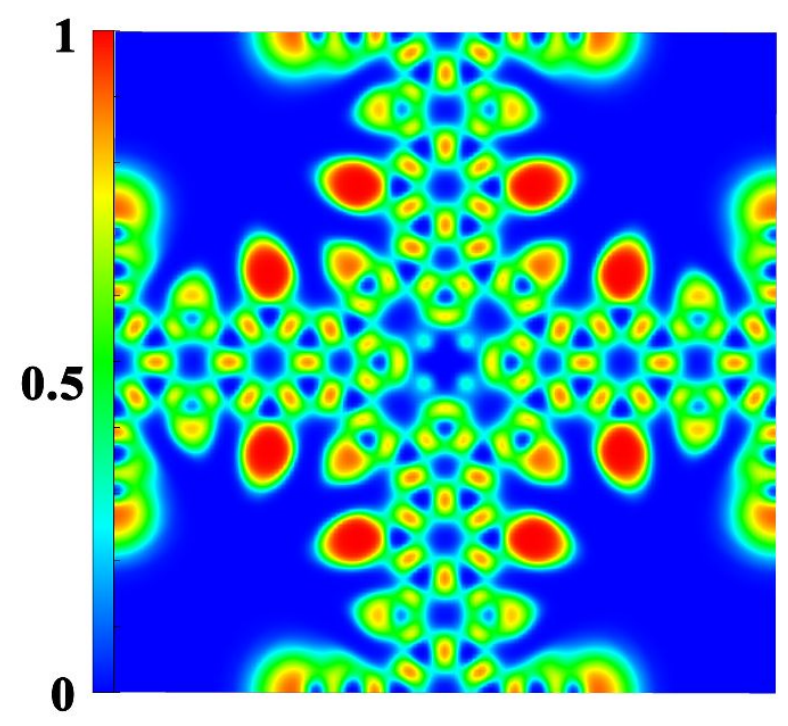

Figure S1. The electron location function (ELF) of the TMPc-TFPN monolayer. The ELF ranges from 0 to 1, corresponding to electron delocalization and accumulation, respectively, while 0.5 indicates an electron-gas-like pair behavior. 


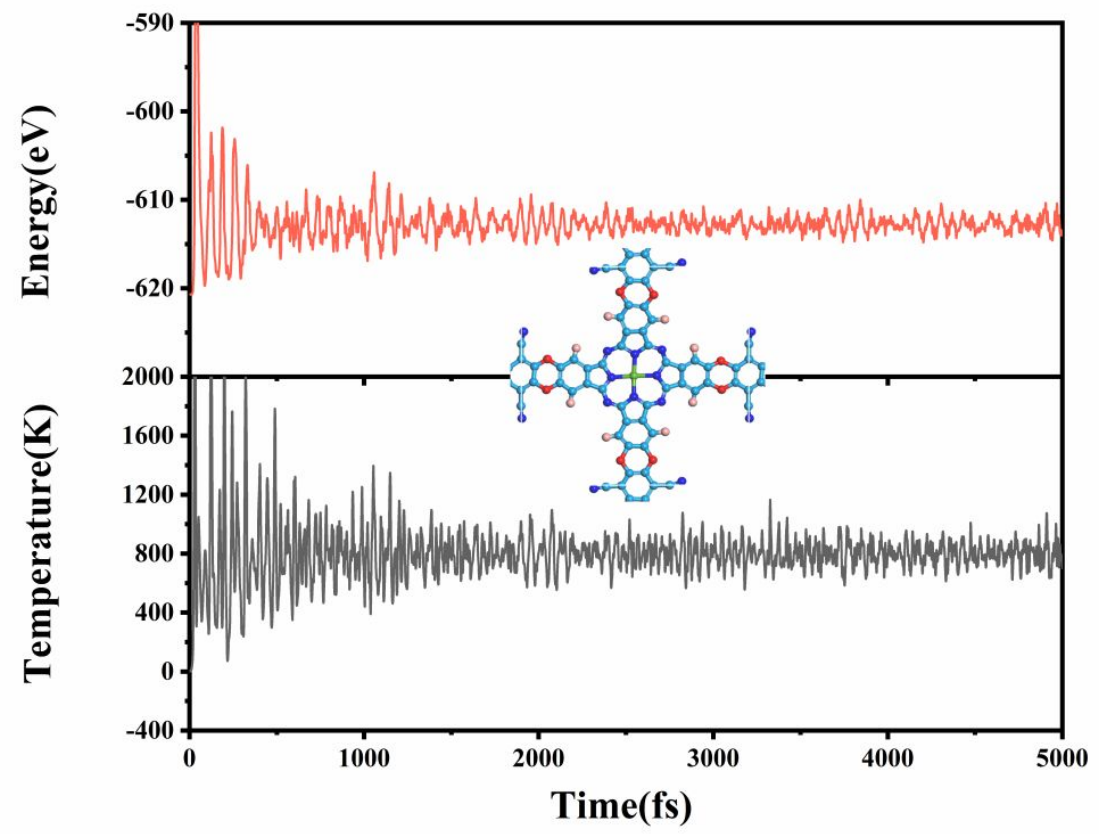

Figure S2. Variations of energy and temperature during 5ps AIMD simulation at $T=$ $800 \mathrm{~K}$ and the inset denotes the top view of WPc-TFPN after AIMD simulation. 

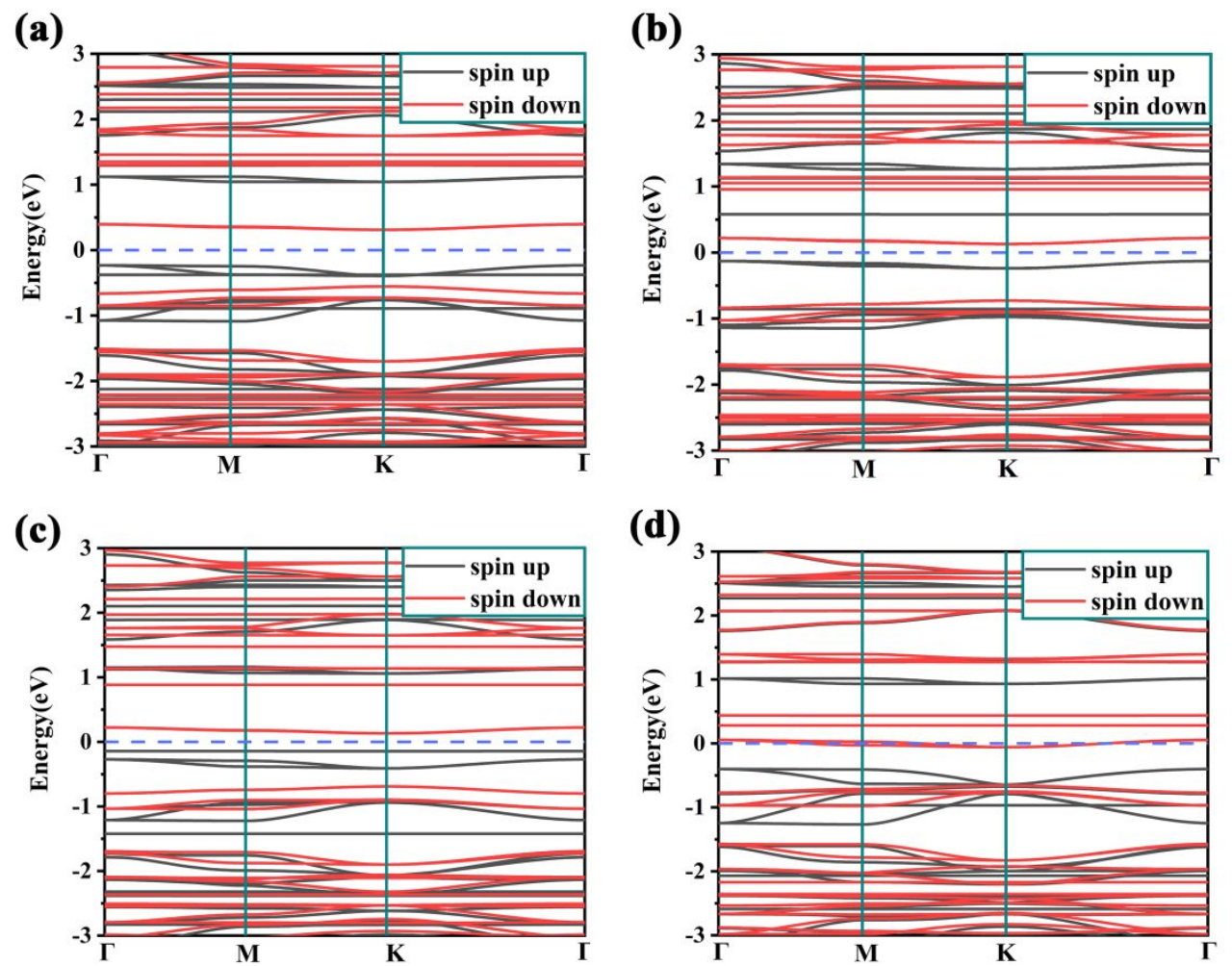

(d)

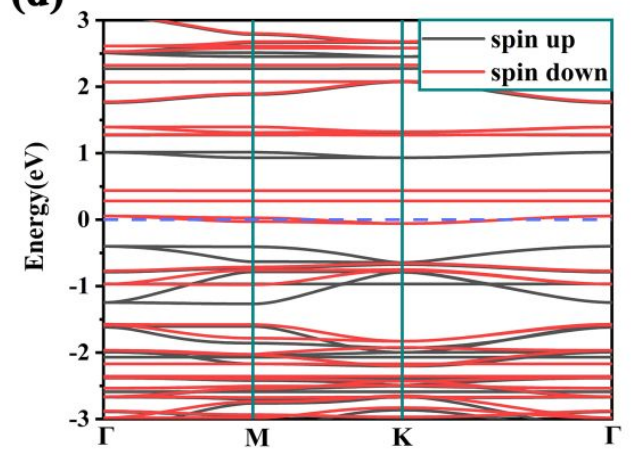

Figure S3. Electronic band structures of (a)Mo, (b)Ta, (c)W and (d) RePc-TFPN at the PBE level. 
(a)

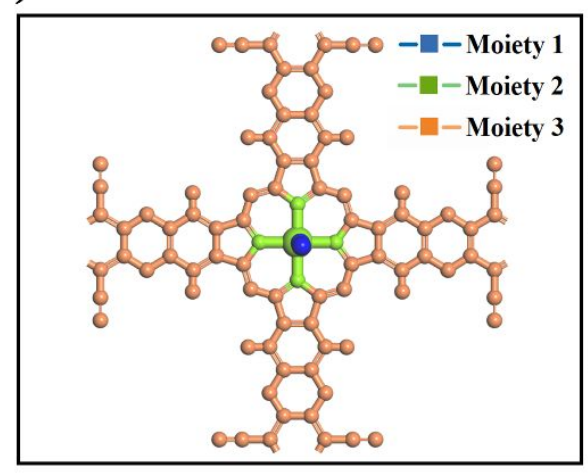

Three Moieties (b)

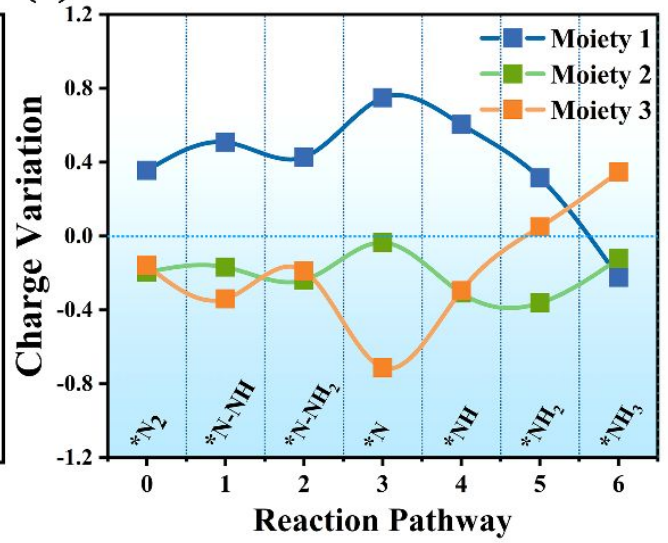

Figure S4. Contributions of different moieties to charge transfer. (a) Division of three different moieties for NxHy-WPc-TFPN systems. Moieties 1, 2 and 3 indicate the * $\mathrm{NxHy}$ (blue), $\mathrm{WN}_{4}$ atom(green) and $\mathrm{COF}$ (orange), respectively. (b)The charge variation of three moieties for the WPc-TFPN along the distal pathway. 


\section{References}

(1) Computational Chemistry Comparison and Benchmark Database. $\underline{\text { http://cccbdb.nist.gov/. }}$

(2) Greeley, J.; Norskov, J. K. Electrochemical Dissolution of Surface Alloys in Acids: Thermodynamic Trends from First-Principles Calculations. Electrochim. Acta 2007, 52, 5829-5836.

(3) Zhao, M.-R.; Song, B.; Yang, L.-M. Two-Dimensional Single-Atom Catalyst $\mathrm{TM}_{3}(\mathrm{HAB})_{2}$ Monolayers for Electrocatalytic Dinitrogen Reduction Using Hierarchical High-Throughput Screening. ACS Appl. Mater. Interfaces 2021, 13, 26109-26122.

(4) Ying, Y. R.; Fan, K.; Luo, X.; Qiao, J. L.; Huang, H. T. Transition MetalTetracyanoquinodimethane Monolayers as Single-Atom Catalysts for the Electrocatalytic Nitrogen Reduction Reaction. Mater. Adv. 2020, 1, 1285-1292.

(5) Wu, Q.; Peng, R.; Huang, B.; Kou, L.; Dai, Y.; Ma, Y. Highly Effective and Selective Molecular Nanowire Catalysts for Hydrogen and Ammonia Synthesis. $J$. Mater. Chem. A 2020, 8, 26075-26084.

(6) Wang, C.; Zhao, Y.-N.; Zhu, C.-Y.; Zhang, M.; Geng, Y.; Li, Y.-G.; Su, Z.-

M. A Two-Dimensional Conductive Mo-Based Covalent Organic Framework as an Efficient Electrocatalyst for Nitrogen Fixation. J. Mater. Chem. A 2020, 8, 2359923606.

(7) Liu, S.; Liu, Y.; Gao, X.; Tan, Y.; Cheng, Z.; Shen, Z.; Fan, M. TwoDimensional Transition Metal Porphyrin Sheets as a Promising Single-Atom-Catalyst for Dinitrogen Electrochemical Reduction to Ammonia: A Theoretical Study. J. Phys. Chem. C 2020, 124, 1492-1499. 
(8) Liu, S.; Liu, Y.; Gao, X.; Tan, Y.; Shen, Z.; Fan, M. First Principle Study of Feasibility of Dinitrogen Reduction to Ammonia on Two-Dimensional Transition Metal Phthalocyanine Monolayer. Appl. Surf. Sci. 2020, 500, 144032.

(9) Liu, F.; Song, L.; Liu, Y.; Zheng, F.; Wang, L.; Palotas, K.; Lin, H.; Li, Y. Using the N-N Dipole as a Theoretical Indicator for Estimating the Electrocatalytic Performance of Active Sites in the Nitrogen Reduction Reaction: Single Transition Metal Atoms Embedded in Two Dimensional Phthalocyanine. J. Mater. Chem. A 2020, 8, 3598-3605.

(10) Huang, C.-X.; Li, G.; Yang, L.-M.; Ganz, E. Ammonia Synthesis Using Single-Atom Catalysts Based on Two-Dimensional Organometallic Metal Phthalocyanine Monolayers under Ambient Conditions. ACS Appl. Mater. Interfaces 2021, 13, 608-621.

(11) Choi, C.; Back, S.; Kim, N.-Y.; Lim, J.; Kim, Y.-H.; Jung, Y. Suppression of Hydrogen Evolution Reaction in Electrochemical $\mathrm{N}_{2}$ Reduction Using Single-Atom Catalysts: A Computational Guideline. ACS Catal. 2018, 8, 7517-7525.

(12) Lv, S.-Y.; Huang, C.-X.; Li, G.; Yang, L.-M. Electrocatalytic Mechanism of $\mathrm{N}_{2}$ Reduction Reaction by Single-Atom Catalyst Rectangular TM-TCNQ Monolayers. ACS Appl. Mater. Interfaces 2021, 13, 29641-29653. 